J. Lake Sci. (湖泊科学), 2019, 31(5): 1299-1309

DOI 10. 18307/2019. 0521

(c) 2019 by Journal of Lake Sciences

\title{
金佛山世界遗产地岩溶地下河系统硝酸盐来源与转化
}

\author{
明晓星, 杨平恒**, 谢世友, 盛 婷, 罗 丹 \\ (西南大学地理科学学院, 岩溶环境重庆市重点实验室, 自然资源部岩溶生态环境-重庆南川野外基地, 重庆 400715)
}

\begin{abstract}
摘 要: 地下水硝酸盐污染已成为一个普遍的环境问题. 为研究重庆金佛山水房泉岩溶地下河系统的硝酸盐来源与转 化, 于 2017 年 4-10月每 $24 \mathrm{~d}$ 左右对地下河系统内的某酒店自来水、化粪池、 $1^{\#}$ 落水洞、水房泉 4 个采样点开展监测, 进 行水化学和 $\delta^{15} \mathrm{~N}_{\text {nitrate }} \delta^{18} \mathrm{O}_{\text {nitrate }}$ 同位素分析. 某酒店污水经化粪池处理后, 由 $1^{\#}$ 落水洞排人地下河, 最后在水房泉排泄. 结果表明: (1)水房泉 $\mathrm{NO}_{3}^{-}$浓度范围为 $4.65 \sim 10.20 \mathrm{mg} / \mathrm{L}$, 相对于我国生活饮用水标准处于较低水平; 化粪池、 $1^{\#}$ 落水洞、水 房泉 3 个采样点电导率和 $\mathrm{NO}_{3}^{-} 、 \mathrm{Cl}^{-}$浓度的高值期与游客人数增多对应关系较好. (2)某酒店自来水 $\delta^{15} \mathrm{~N}_{\text {nitrate }}$ 值为 $3.7 \%$ $5.8 \% 0 、 \delta^{18} \mathrm{O}_{\text {nitrate }}$ 值为 $1.6 \% \sim 2.7 \%$, 说明硝酸盐主要来源为土壤有机氮, 处于自然背景值; $1^{\#}$ 落水洞 $\delta^{15} \mathrm{~N}_{\text {nitrate }}$ 值为 $14.4 \% 0 \sim$ $21.1 \% 0 、 \delta^{18} \mathrm{O}_{\text {nitrate }}$ 值为 $3.5 \% 0 \sim 11.2 \%$, 显示硝酸盐主要来源为粪肥污水; 化粪池和水房泉的 $\delta^{15} \mathrm{~N}_{\text {nitrate }}$ 值为 $3.7 \% 0 \sim 17.0 \%$ 、 $\delta^{18} \mathrm{O}_{\text {nitrate }}$ 值为 $-9.0 \% 0 \sim 7.3 \%$, 表明硝酸盐主要来源为土壤有机氮与粪肥污水, 显示其硝酸盐主要污染源是酒店生活废污 水. (3)某酒店自来水、水房泉地下水的硝酸盐转化过程以同化作用为主; 化粪池污水以硝化作用为主, 是岩溶地下河系统 硝酸盐的重要来源之一; $1^{\#}$ 落水洞污水表现为反硝化作用. (4)基于 SIAR 模型对水房泉的硝酸盐来源进行定量解析, 发现 大气降水、土壤有机氮和粪肥污水的贡献率分别为 $28 \%$ 、36\% 和 $36 \%$ 左右.
\end{abstract}

关键词：地下河;硝酸盐;氮氧同位素;来源与转化;SIAR 模型;金佛山

\section{Sources and transformations of nitrate of the subterranean river system in Jinfoshan Karst World Heritage}

MING Xiaoxing, YANG Pingheng ** , XIE Shiyou, SHENG Ting \& LUO Dan

(School of Geographical Sciences, Southwest University, Chongqing Key Laboratory of Karst Environment, Field Scientific Observation \& Research Base of Karst Eco-environments at Nanchuan in Chongqing, Ministry of Nature Resources of the People's Republic of China, Chongqing 400715, P.R. China)

Abstract: Nitrate pollution in groundwater has become a common environmental problem. From April to October in 2017, four sampling sites (a hotel's tap water, septic system, Sinkhole $1^{\#}$ and Shuifang spring) in Jinfoshan Karst, Chongqing were monitored every 24 days to investigate the sources and transformations of nitrate. The hydrochemistry and $\delta^{15} \mathrm{~N}_{\text {nitrate }}, \delta^{18} \mathrm{O}_{\text {nitrate }}$ isotopic analyses were carried out. The sewage from hotel was degraded in the septic system, then discharged into the subterranean river by the Sinkhole $1^{\#}$, and finally drained at Shuifang spring. Results showed that the concentrations of nitrate ranged from 4.65 to 10.20 $\mathrm{mg} / \mathrm{L}$ at Shuifang spring, which was at a low level comparing with the standard of drinking water in China. The three high-value periods of the electrical conductivity and concentrations of nitrate and chloride at septic system, Sinkhole $1^{\#}$ and Shuifang spring corresponded well to the increase in the number of tourists. The isotopic nitrate compositions of hotel's tap water were found to range from $3.7 \%$ to $5.8 \%$ for $\delta^{15} \mathrm{~N}_{\text {nitrate }}$ and from $1.6 \%$ to $2.7 \%$ for $\delta^{18} \mathrm{O}_{\text {nitrate }}$, indicating that the nitrate mainly originated from soil organic nitrogen maintaining at a natural background value. The $\delta{ }^{15} \mathrm{~N}_{\text {nitrate }}$ and $\delta^{18} \mathrm{O}_{\text {nitrate }}$ at Sinkhole $1^{\#}$ varied from $14.4 \%$ to $21.1 \%$, and from $3.5 \%$ to $11.2 \%$, respectively, revealing that manure $\&$ sewage was the main source of nitrate at Sinkhole $1^{\#}$. The $\delta{ }^{15} \mathrm{~N}_{\text {nitrate }}$ and $\delta^{18} \mathrm{O}_{\text {nitrate }}$ at septic system and Shuifang spring varied between $3.7 \%$ and $17 \%$ for $\delta^{15} \mathrm{~N}_{\text {nitrate }}$ value, and $-9.0 \%$ and $7.3 \%$ for $\delta^{18} \mathrm{O}_{\text {nitrate }}$ value, suggesting that soil organic nitrogen and manure \& sewage were the major sources of nitrate which was

* 国家自然科学基金项目 (41103068) 和中央高校基本科研业务费专项 (XDJK2018AB002) 联合资助. 2018-11-23 收 稿; 2019-02-27 收修改稿. 明晓星(1994 ), 男,硕士研究生;E-mail : xiaoxingming_swu@ 126.com.

** 通信作者;E-mail:pinghengyang@ 126.com. 
dominantly derived from the domestic sewage of the hotel. In hotel's tap water and groundwater from Shuifang spring, assimilation is the main process of nitrate transformation. While effluent in septic system, one of the important sources of nitrate in the subterranean river system was affected by nitrification. The wastewater at Sinkhole $1^{\#}$ was characterized by denitrification. A SIAR model was used to estimate the contribution of each nitrate source to total nitrate at Shuifang spring, which indicated that the contribution rate of atmospheric precipitation, soil organic nitrogen and manure \& sewage were roughly $28 \%$, $36 \%$ and $36 \%$, respectively.

Keywords: Subterranean river; nitrate; dual isotopes of nitrogen and oxygen; sources and transformations; SIAR model; Jinfoshan

近年来, 随着社会经济的不断发展, 城市 ${ }^{[1]}$ 、农业 ${ }^{[2]}$ 、旅游活动 ${ }^{[3]}$ 对水质的影响越来越大. 硝酸盐浓度过 高是水质恶化的重要表现之一, 并且已成为世界性的环境问题 ${ }^{[4-5]}$. 水中浓度过高的硝酸盐进人人体后被还 原成亚硝酸盐, 会引发婴儿的高铁血红蛋白症、胃癌等疾病 ${ }^{[6-7]}$. 因此, 及时识别硝酸盐来源与转化过程并进 行有效管理, 对于改善水质、促进水生态系统的良性循环起着至关重要的作用. 早期, 单独使用 $\delta^{15} \mathrm{~N}_{\text {nitrate }}$ 特征 值识别污染源 ${ }^{88]}$, 但是 $\delta^{15} \mathrm{~N}_{\text {nitrate }}$ 在运移、混合中受硝化 $\left(\mathrm{NH}_{4}^{+}\right.$通过自养型微生物氧化为 $\mathrm{NO}_{3}^{-}$的过程)、反硝化 $\left(\mathrm{NO}_{3}^{-}\right.$通过微生物还原为气态氮 $\mathrm{N}_{2} 、 \mathrm{~N}_{2} \mathrm{O}$ 的过程)、同化 $\left(\mathrm{NH}_{4}^{+} 、 \mathrm{NO}_{3}^{-} 、 \mathrm{~N}_{2} \mathrm{O} 、 \mathrm{~N}_{2}\right.$ 通过微生物和植物吸收同化) 等 作用的影响, 从而造成同位素分馏和特征值改变, 无法准确、清晰地划分来源 ${ }^{[9-10]}$. 随着同位素检测技术的发 展, $\delta^{18} \mathrm{O}_{\text {nitrate }}$ 也能够被同时测定, 可有效地辅助硝酸盐来源识别与转化研究 ${ }^{[11]}$. 综合利用水化学、 $\delta^{15} \mathrm{~N}_{\text {nitrate }}$ 、 $\delta^{18} \mathrm{O}_{\text {nitrate }}$ 和模型解译来源贡献率等方法, 可识别不同地区的硝酸盐来源与转化 ${ }^{[2-3,12-16]}$, 为控制硝酸盐输人提 供科学依据.

岩溶含水层极其特殊, 主要表现为三维空间上的非均质性和水力联系的各向异性 ${ }^{[17]}$, 这些特征决定了 其一旦遭受硝酸盐污染将极难治理. 因此, 进一步明确岩溶区硝酸盐的动态变化特征、来源和转化机理, 这

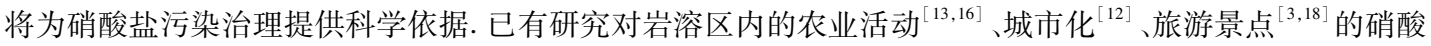
盐来源进行了识别, 其中旅游活动引起的岩溶地下水硝酸盐污染受到的关注最少. 金佛山分别于 2013 年、 2014 年被列为国家 5A 级景区和世界遗产地, 其已成为游客春季赏花、夏季避暑、秋季观景、冬季滑雪的胜 地. 据不完全统计, 2017 年金佛山景区游客达 67 万人 ${ }^{[19]}$. 伍坤宇等 ${ }^{[20]}$ 利用地球化学 $\left(\mathrm{NO}_{3}^{-}-\mathrm{N}\right.$ 浓度 $)$ 、统计学 和 GIS 相结合的方法, 研究金佛山地区地下水 $\mathrm{NO}_{3}^{-}-\mathrm{N}$ 浓度的时空变异特征, 发现来自旅游业的压力导致 $\mathrm{NO}_{3}^{-}-\mathrm{N}$ 浓度总体呈上升趋势. Yang 等 ${ }^{[3,18]}$ 利用 $\delta^{15} \mathrm{~N}_{\text {nitrate }}$ 和 $\delta^{18} \mathrm{O}_{\text {nitrate }}$ 研究金佛山世界遗产地内岩溶地下水系 统中硝酸盐的来源, 发现酒店废污水是主要污染源. 然而, 目前尚未开展金佛山世界遗产地内岩溶地下水系 统中硝酸盐的转化过程研究, 这对于更好地防治硝酸盐污染是非常不利的. 因此, 开展该地区硝酸盐来源与 转化的定量识别研究显得具有重要的理论与现实意义. 基于此, 本文基于水化学和 $\delta^{15} \mathrm{~N}_{\text {nitrate }} \delta^{18} \mathrm{O}_{\text {nitrate }}$ 开展金 佛山世界遗产地内硝酸盐来源与转化研究, 并运用 SIAR 模型定量分析不同来源硝酸盐的贡献率, 可为控制 遗产地内硝酸盐的输人提供建议与科学依据.

\section{1 研究区概况}

金佛山世界遗产地 $\left(28^{\circ} 49^{\prime} 37^{\prime \prime} \sim 29^{\circ} 11^{\prime} 30^{\prime \prime} \mathrm{N}, 107^{\circ} 00^{\prime} 56^{\prime \prime} \sim 107^{\circ} 25^{\prime} 34^{\prime \prime} \mathrm{E}\right)$ 地处大娄山北端, 四川盆地东南 缘与云贵高原的过渡地带, 主峰风吹岭海拔 $2251 \mathrm{~m}$, 总面积 $41850 \mathrm{hm}^{2}$, 属典型的岩溶地貌 ${ }^{[21]}$. 在地质构造 上, 金佛山属于一个宽缓的向斜, 轴部为二叠系碳酸盐岩地层, 形成了海拔 $2100 \mathrm{~m}$ 左右具有较大面积的缓 坡和平台, 发育有大型的岩溶洼地、落水洞、洞穴系统等岩溶形态 ${ }^{[22]}$. 受山体较大相对高差的影响, 金佛山 产生了垂直气候分带, 山体上部具有温带湿润气候的某些特征, 云雾多、日照少、雨量丰沛, 空气湿度大, 多 年平均气温、降水量分别为 $8.2^{\circ} \mathrm{C} 、 1434.5 \mathrm{~mm}$,降水集中分布于 $4-10$ 月 ${ }^{[22]}$.

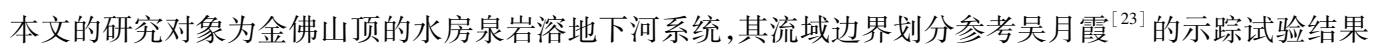
(图 1). 流域内分布着一个呈 $\mathrm{NE} \sim \mathrm{SW}$ 向的药池坝洼地, 洼地底部相对较为平坦 ${ }^{[23]}$. 洼地边缘落水洞发育, 密度约为 $6 \sim 7$ 个 $/ \mathrm{km}^{2}$, 这些落水洞是降水形成径流往地下汇流的人口 ${ }^{[23]}$. 流域地下水主要从山顶西坡陡崖 上的水房泉排泄, 其多年平均流量为 $12.6 \times 10^{4} \sim 15.8 \times 10^{4} \mathrm{~m}^{3} / \mathrm{a}^{[23-24]}$.

因旅游需要, 山顶核心区药池坝洼地建设有某酒店, 其污水经管道进人化粪池沉淀、降解之后, 滤液通 
过洼地西部的 $1^{\#}$ 落水洞排人岩溶含水层, 最后在水房泉排泄 ${ }^{[24]}$ (图 1). 苂光剂示踪试验和 $\delta D_{\text {water }} \delta \delta^{18} O_{\text {water }}$ 同 位素方法已证明酒店污水通过 $1^{\#}$ 落水洞对岩溶地下河造成污染, 污水从酒店运移至水房泉最快仅需 $26.8 \mathrm{~h}$, 最大影响时间可达 $56.2 \mathrm{~d}^{[25]}$. 化粪池的体积约为 $120 \mathrm{~m}^{3}$, 污水为自然排放, 排放量不定, 旅游旺季排放量大, 排泄量平均值约为 $0.6 \mathrm{~L} / \mathrm{s}^{[25]}$.

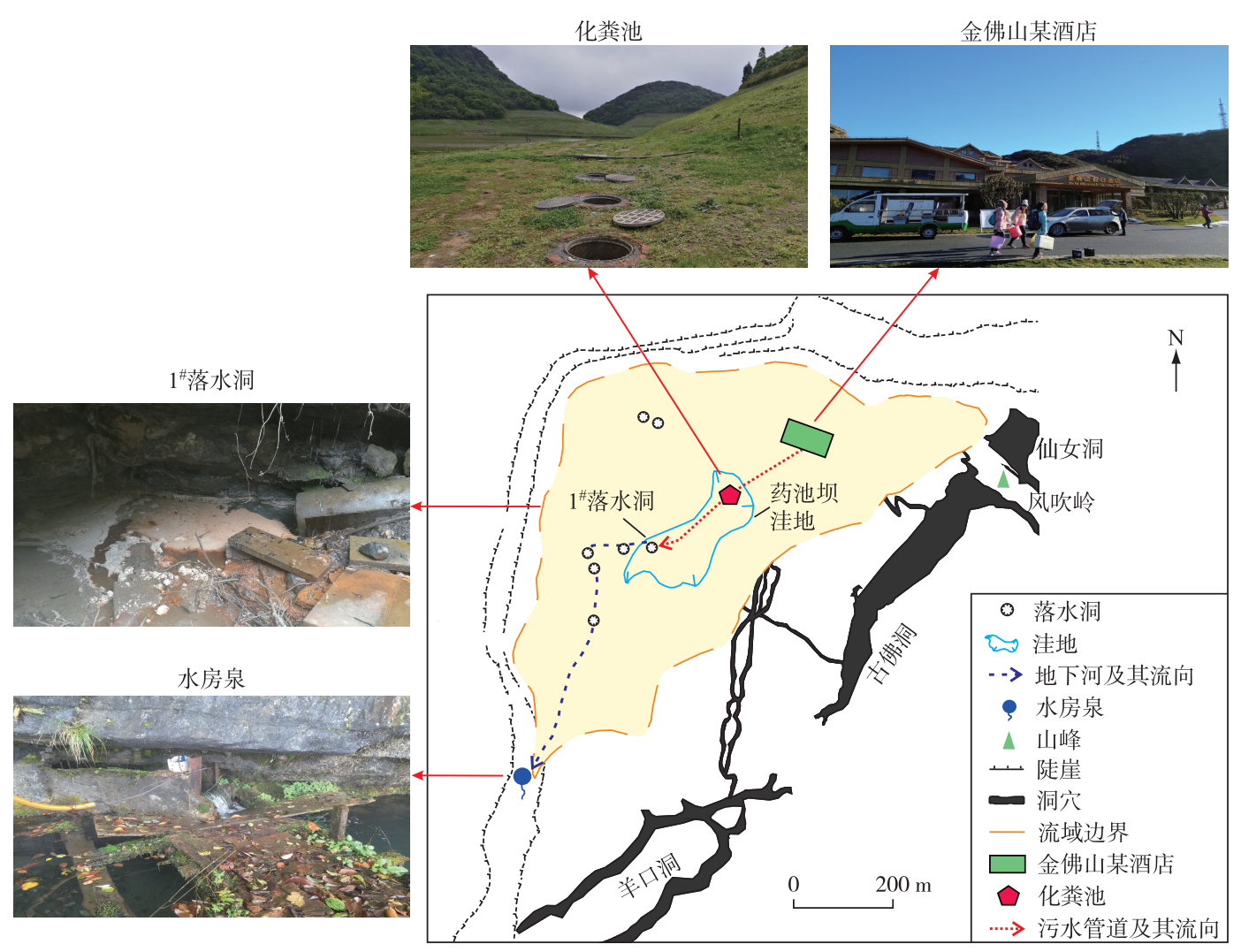

图 1 研究区位置及采样点分布

Fig.1 Location and sampling sites distribution within the study area

\section{2 研究方法}

\section{1 野外采样}

水房泉岩溶地下河系统共设 4 个采样点,分别为某酒店内的自来水 (来源于金佛山东北部的仙女洞)、 化粪池、 $1^{\#}$ 落水洞、水房泉 (图 1), 采样时间为 2017 年 4-10 月, 取样间隔为 $24 \mathrm{~d}$ 左右 1 次,共 24 个样品. 所 有采样点现场使用 Multi3430 便携式多参数水质分析仪 (德国 WTW 公司), 测定水温 ( T)、 $\mathrm{pH}$ 值、电导率 (EC)、溶解氧 (DO) 4 项指标,测量精度分别为 $0.1^{\circ} \mathrm{C} 、 0.01 、 1 \mu \mathrm{S} / \mathrm{cm} 、 0.01 \mathrm{mg} / \mathrm{L}$.

用于测试 $\delta^{15} \mathrm{~N}_{\text {nitrate }} \delta \delta^{18} \mathrm{O}_{\text {nirrate }}$ 的样品在采样前, 用直径 $50 \mathrm{~mm}$ 、孔径 $0.22 \mu \mathrm{m}$ 的混合纤维素膜过滤待采水 样取 $40 \mathrm{ml}$, 装于洗净的聚乙烯瓶中冷藏运回实验室. 取 $550 \mathrm{ml}$ 水样装于洁净的聚乙烯瓶中, 确保瓶内无气 泡,用于检测阴离子与 $\mathrm{NH}_{4}^{+}$浓度. 为尽量减小误差,所有样品采样前先用待采水样润洗样瓶 3 次.

\section{2 样品测试}

$\mathrm{NO}_{3}^{-}$浓度检测参照饮用天然矿泉水检测方法 ( GB/T 8538- 2008), 测试仪器为紫外分光光度计 (UV2450, 日本岛津公司) ; $\mathrm{Cl}^{-}$浓度测定采用 $\mathrm{AgNO}_{3}$ 滴定法; $\mathrm{NH}_{4}^{+}$浓度测定采用 $\mathrm{HQ} 40 \mathrm{~d}$ 多参数水质分析仪 (美国 HACH), 精度为 $0.01 \mathrm{mg} / \mathrm{L}$. 以上样品测试均在岩溶环境重庆市重点实验室分析完成. $\delta^{15} \mathrm{~N}_{\text {nitrate }}$ 、 
$\delta^{18} \mathrm{O}_{\text {nitrate }}$ 在中国农业科学院农业环境稳定同位素实验室进行测试分析, 主要利用特异性的反硝化细菌将 $\mathrm{NO}_{3}^{-}$转化为 $\mathrm{N}_{2} \mathrm{O}$, 再采用 ISOPRIME100-Tracegas 痕量气体一同位素质谱连用仪(英国 Isoprime 公司) 完成 $\mathrm{N}_{2} \mathrm{O}$ 的 $N 、 O$ 同位素测定, 其中 $\mathrm{N}$ 同位素以标准大气中的 $\mathrm{N}_{2}$ 为参照, $\mathrm{O}$ 同位素以维也纳标准平均海水 ( V-SMOW) 为参照, 采用 USGS32 $\left(\delta^{15} \mathrm{~N}=180 \%, \delta^{18} \mathrm{O}=25.7 \%\right.$ ) 、USGS34 $\left(\delta^{15} \mathrm{~N}=-1.8 \%, \delta^{18} \mathrm{O}=-27.9 \%\right.$ )、USGS35 $\left(\delta^{15} \mathrm{~N}=\right.$ $2.7 \%{ }_{0}, \delta^{18} \mathrm{O}=57.5 \%$ ) 为标准, 用两点校正的方程对所测定的气体进行校正, 检测精度为 $0.01 \%, \delta^{15} \mathrm{~N}_{\text {nitrate }}$ 测定 结果的相对标准偏差 $\leqslant 0.39 \%, \delta^{18} \mathrm{O}_{\text {nitrate }}$ 测定结果的相对标准偏差 $\leqslant 0.21 \%$.

\subsection{SIAR 稳定同位素混合模型}

SIAR (Stable Isotope Analysis in R) 模型是一个稳定同位素混合模型, 由 Parnell 等 ${ }^{[26]}$ 开发和应用. 在 $R$ 语言库中运行 SIAR 模型程序包并将所需数据输人进行计算与出图. 为了确定一系列 $N$ 个混合物中 $J$ 同位 素的 $K$ 来源贡献率,SIAR 混合模型可如式 (1) 所示 ${ }^{[5]}$ :

$$
\begin{aligned}
X_{i j}= & \sum_{k=1}^{K} P_{k}\left(S_{j k}+C_{j k}\right)+\varepsilon_{i j} \\
S_{j k} & \sim N\left(\mu_{j k}, \omega_{j k}^{2}\right) \\
C_{j k} & \sim N\left(\lambda_{j k}, \tau_{j k}^{2}\right) \\
\varepsilon_{i j} & \sim N\left(0, \sigma_{j}^{2}\right)
\end{aligned}
$$

式中, $X_{i j}$ 是混合物 $i$ 中同位素 $j$ 的值, $i=1 、 2 、 3 、 \ldots 、 N, j=1 、 2 、 3 、 \ldots 、 J ; P_{k}$ 是来源 $k$ 的比例, 其需要通过 SIAR 模 型进行评价; $S_{j k}$ 是来源 $k$ 中同位素 $j$ 的值 $(k=1 、 2 、 3 、 \ldots 、 K)$, 其平均值 $\mu_{j k}$ 和标准偏差 $\omega_{j k}$ 呈正态分布; $C_{j k}$ 是 来源 $k$ 中同位素 $j$ 的分馏系数, 其平均值 $\lambda_{j k}$ 和标准偏差 $\tau_{j k}$ 呈正态分布; $\varepsilon_{i j}$ 是残留误差, 代表个别混合物间 额外无法定量的变化,其平均值 0 和标准偏差 $\sigma_{j}$ 呈正态分布.

\section{3 结果与讨论}

\section{1 硝酸盐的时空变化特征}

2017 年 4- 10 月研究区 4 个采样点 $\mathrm{NO}_{3}^{-}$浓度的变化范围为 $0.96 \sim 40.79 \mathrm{mg} / \mathrm{L}($ 表 1 ), 平均值为 11.95 $\mathrm{mg} / \mathrm{L}$. 其中, 化粪池的 $\mathrm{NO}_{3}^{-}$浓度呈现出明显的时间变化, 高值主要出现在 4 月和 7-9 月. 此外, $\mathrm{EC}$ 值和 $\mathrm{Cl}^{-}$ 浓度也有类似趋势, 这与金佛山景区游客人数相关(图 2).

某酒店自来水 $\mathrm{DO}$ 浓度为 $8.05 \sim 8.98 \mathrm{mg} / \mathrm{L}\left(\right.$ 表 1), $\mathrm{NO}_{3}^{-} 、 \mathrm{NH}_{4}^{+} 、 \mathrm{Cl}^{-}$浓度总体较低(图 2), 表明其水质较好.

化粪池污水 $\mathrm{EC}$ 值为 $268 \sim 743 \mu \mathrm{S} / \mathrm{cm}$ (表 1), 4 月和 7-9 月 EC 值较高, 同期 $\mathrm{NO}_{3}^{-} 、 \mathrm{Cl}^{-}$浓度有升高趋势 (图 2). 其主要原因是 4 月金佛山杜鹃花盛开,有大量游客上山观赏; 7-8 月重庆地区连续高温, 上山避暑 的游客增多; 9 月中下旬至十一黄金周期间金佛山赏秋景的游客较多 (图 2), 从而导致酒店排污量增加. 值 得注意的是, 5 月 25 日 DO 浓度降至 $1.42 \mathrm{mg} / \mathrm{L}, \mathrm{NO}_{3}^{-}$浓度降至 $1.12 \mathrm{mg} / \mathrm{L}, \mathrm{Cl}^{-}$浓度与 4 月 29 日的相比有所下 降但趋势略和缓, 而 $\mathrm{NH}_{4}^{+}$浓度略有上升 (图 2). 有研究表明, $\mathrm{DO}$ 浓度 $<0.2 \mathrm{mg} / \mathrm{L}$ 条件下反硝化速率最为理 想, DO 浓度介于 2 6 mg/L的条件下仍有反硝化作用存在, 但速率最小 ${ }^{[27]}$. 因此, 5 月 25 日的化粪池污水可 能出现反硝化作用, 导致 $\mathrm{NO}_{3}^{-}$浓度偏低.

$1^{\#}$ 落水洞 $\mathrm{EC}$ 值为 $273 \sim 401 \mu \mathrm{S} / \mathrm{cm}, \mathrm{NO}_{3}^{-}$浓度为 $0.96 \sim 2.19 \mathrm{mg} / \mathrm{L}, \mathrm{NH}_{4}^{+}$浓度为 $3.46 \sim 7.33 \mathrm{mg} / \mathrm{L}($ 表 1$)$. 一 方面, 对比 4 月 29 日和 5 月 25 日的 $\mathrm{EC}$ 值、 $\mathrm{NH}_{4}^{+}$和 $\mathrm{Cl}^{-}$浓度, 发现 5 月 25 日样品的污染水平有所降低, 主要 原因是景区北坡索道检修关闭导致到达研究区的游客数量减少. 另一方面,有研究表明 DO 浓度为 $2 \sim 6 \mathrm{mg} / \mathrm{L}$ 时仍可能发生反硝化作用, 只是速率最小 ${ }^{[27]}$, 而 $\mathrm{pH}$ 值介于 $7.0 \sim 8.0$ 之间则是反硝化作用发生的最佳范 围 ${ }^{[28]} .4$ 月 29 日和 5 月 25 日的 DO 浓度分别为 6.55 和 $6.53 \mathrm{mg} / \mathrm{L}, \mathrm{pH}$ 值分别为 7.66 和 $7.34, \mathrm{NO}_{3}^{-}$浓度都偏 低, 表明反硝化作用发生. 综上所述, 污水由化粪池经管道运移至 $1^{\#}$ 落水洞的过程中, 主要发生反硝化作用, 而硝化作用受到抑制.

水房泉 DO 浓度为 7.60 9.00 mg/L; EC 值为 $215 \sim 290 \mu \mathrm{S} / \mathrm{cm}$ (表 1), 其中最高值出现在 8 月 7 日; 同期 $\mathrm{NO}_{3}^{-}$与 $\mathrm{Cl}^{-}$浓度出现监测期间的相对高值, 主要是由于暑期游客人数增长导致酒店排污量增大 (图 2). 此外, 对比酒店自来水与水房泉 $\mathrm{NO}_{3}^{-}$浓度, 发现两处 $\mathrm{NO}_{3}^{-}$浓度极其相似, 只有夏季旅游高峰时水房泉 $\mathrm{NO}_{3}^{-}$浓度略 
有上升 (图 2), 表明这两个点位可能有同样的硝酸盐来源与转化过程.

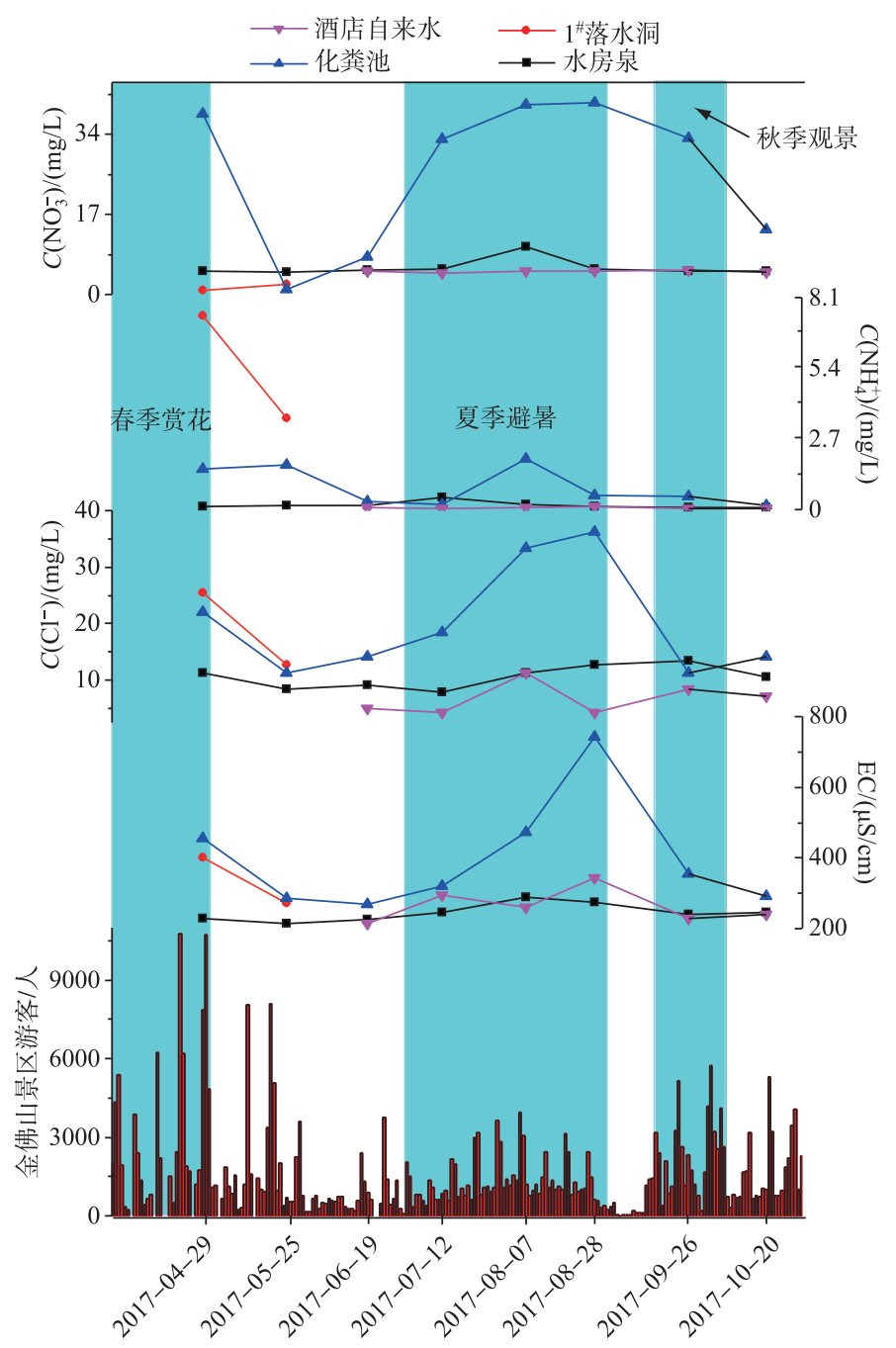

图 2 研究区水化学指标的时间变化

Fig. 2 Temporal variation of the hydrochemical index in the study area

\section{2 硝酸盐的来源与转化}

3.2.1 硝酸盐的来源 据前人对硝酸盐来源区间划分的研究成果 ${ }^{[15,29-32]}, \delta^{15} \mathrm{~N}_{\text {nitrate }}$ 变化范围为: 大气沉降为 $-10 \% 0 \sim 13 \%$, 土壤有机氮为 $0 \sim 8 \%$, 粪肥污水为 $6 \%{ }_{0} \sim 25 \%, \mathrm{NO}_{3}^{-}$化肥为 $-4 \% 0 \sim 7 \%$, 肥料中的 $\mathrm{NH}_{4}^{+}$为 $-6 \%{ }_{0} \sim$ $6 \% ; \delta^{18} \mathrm{O}_{\text {nitrate }}$ 变化范围为: 大气沉降为 $25 \% 0 \sim 75 \%$, 土壤有机氮、肥料中的 $\mathrm{NH}_{4}^{+}$、粪肥污水为 $-5 \% 0 \sim 15 \%, \mathrm{NO}_{3}^{-}$ 化肥为 $15 \%$ $25 \%$. 本研究所有样品 $\delta^{15} \mathrm{~N}_{\text {nitrate }}$ 波动区间为 $3.7 \%$ 21.1\% , 均值为 $7.5 \%$; $\delta^{18} \mathrm{O}_{\text {nirate }}$ 波动区间为 $-9.0 \% 0 \sim 11.2 \%$, 均值为 $0.1 \% 0$ (表 1). 将所有样品的 $\delta^{15} \mathrm{~N}_{\text {nitrat }}, \delta^{18} \mathrm{O}_{\text {nitrate }}$ 数据投射到 $\delta^{15} \mathrm{~N}_{\text {nitrate }} 、 \delta^{18} \mathrm{O}_{\text {nitrate }}$ 来源分 布图中区分硝酸盐来源可以发现, 水房泉岩溶地下河系统硝酸盐主要来源为土壤有机氮和粪肥污水 (图 3 ).

某酒店自来水样品分布在土壤有机氮与肥料中的 $\mathrm{NH}_{4}^{+}$范围内 (图 3). 由于金佛山世界遗产地内无农业 活动, 同时该点水源地周围无人类活动, 因此该点硝酸盐主要来自土壤有机氮且 $\delta^{15} \mathrm{~N}_{\text {nitrate }}$ 和 $\delta^{18} \mathrm{O}_{\text {nitrate }}$ 处于自 然背景值. 
表 12017 年 4-10 月研究区水化学指标及氮氧同位素

Tab.1 Hydrochemical index and isotopic compositions of nitrogen and oxygen in the study area from April to October, 2017

\begin{tabular}{|c|c|c|c|c|c|c|c|c|c|c|}
\hline 采样点 & 日期 & EC & $\mathrm{pH}$ 值 & DO & $\mathrm{T}$ & $\mathrm{NO}_{3}^{-}$ & $\mathrm{Cl}^{-}$ & $\mathrm{NH}_{4}^{+}$ & $\delta^{15} \mathrm{~N}_{\text {nitrate }}$ & $\delta^{18} \mathrm{O}_{\text {nitrate }}$ \\
\hline \multirow[t]{6}{*}{ 酒店自来水 } & $2017-06-19$ & 214 & 6.99 & 8.34 & 13.5 & 4.86 & 4.96 & 0.09 & 4.1 & 2.1 \\
\hline & $2017-07-12$ & 294 & 7.92 & 8.05 & 15.0 & 4.53 & 4.25 & 0.04 & 5.8 & 2.4 \\
\hline & $2017-08-07$ & 261 & 7.60 & 8.47 & 18.0 & 5.02 & 11.34 & 0.09 & 3.8 & 2.7 \\
\hline & $2017-08-28$ & 344 & 8.08 & 8.19 & 18.2 & 5.03 & 4.25 & 0.13 & 3.7 & 2.0 \\
\hline & $2017-09-26$ & 230 & 8.04 & 8.98 & 17.3 & 5.09 & 8.51 & 0.05 & 4.9 & 1.7 \\
\hline & $2017-10-20$ & 239 & 8.23 & 8.98 & 12.3 & 4.74 & 7.09 & 0.04 & 3.7 & 1.6 \\
\hline \multirow[t]{8}{*}{ 化粪池 } & $2017-04-29$ & 456 & 8.37 & 4.59 & 9.1 & 38.43 & 21.98 & 1.52 & 9.3 & -1.0 \\
\hline & $2017-05-25$ & 285 & 7.37 & 1.42 & 11.3 & 1.12 & 11.34 & 1.69 & - & - \\
\hline & $2017-06-19$ & 268 & 7.00 & 6.84 & 13.0 & 8.03 & 14.18 & 0.31 & 7.2 & -6.0 \\
\hline & $2017-07-12$ & 321 & 7.49 & 6.22 & 13.7 & 32.92 & 18.43 & 0.18 & 3.7 & -6.8 \\
\hline & $2017-08-07$ & 474 & 7.02 & 4.40 & 18.5 & 40.28 & 33.32 & 1.90 & 5.7 & -5.6 \\
\hline & $2017-08-28$ & 743 & 7.64 & 3.80 & 20.1 & 40.79 & 36.16 & 0.52 & 5.0 & -7.4 \\
\hline & $2017-09-26$ & 355 & 7.82 & 6.25 & 17.0 & 33.24 & 11.34 & 0.48 & 4.3 & -7.8 \\
\hline & $2017-10-20$ & 292 & 7.87 & 6.35 & 14.0 & 13.80 & 14.18 & 0.15 & 6.1 & -9.0 \\
\hline \multirow[t]{2}{*}{$1^{\#}$ 落水洞 } & $2017-04-29$ & 401 & 7.66 & 6.55 & 9.3 & 0.96 & 25.52 & 7.33 & 21.1 & 11.2 \\
\hline & $2017-05-25$ & 273 & 7.34 & 6.53 & 11.6 & 2.19 & 12.76 & 3.46 & 14.4 & 3.5 \\
\hline \multirow[t]{8}{*}{ 水房泉 } & $2017-04-29$ & 230 & 8.35 & 8.89 & 10.3 & 4.88 & 11.34 & 0.11 & 17.0 & 3.8 \\
\hline & $2017-05-25$ & 215 & 7.89 & 9.00 & 10.2 & 4.65 & 8.51 & 0.15 & 8.4 & 7.3 \\
\hline & $2017-06-19$ & 227 & 7.01 & 8.58 & 11.0 & 5.25 & 9.22 & 0.17 & 7.3 & 3.4 \\
\hline & $2017-07-12$ & 247 & 7.68 & 8.90 & 10.1 & 5.38 & 7.80 & 0.47 & 7.6 & 2.6 \\
\hline & $2017-08-07$ & 290 & 7.49 & 8.70 & 10.6 & 10.20 & 11.34 & 0.18 & 5.3 & -3.1 \\
\hline & $2017-08-28$ & 274 & 7.86 & 7.60 & 13.0 & 5.46 & 12.76 & 0.11 & 10.3 & 2.7 \\
\hline & $2017-09-26$ & 239 & 7.49 & 8.92 & 10.2 & 4.90 & 13.47 & 0.07 & - & - \\
\hline & $2017-10-20$ & 247 & 8.09 & 8.55 & 10.2 & 5.02 & 10.64 & 0.06 & 7.1 & 1.6 \\
\hline
\end{tabular}

1 ) “一” 表示未检测; 2) $\mathrm{EC}$ 单位为 $\mu \mathrm{S} / \mathrm{cm}, \mathrm{pH}$ 值无单位, $T$ 单位为 ${ }^{\circ} \mathrm{C}, \delta^{15} \mathrm{~N}_{\text {nitrate }} \delta^{18} \mathrm{O}_{\text {nitrate }}$ 以\%o计,其他指标单位为 $\mathrm{mg} / \mathrm{L}$.

总体来看, 化粪池样品大部分分布在土壤有机氮与粪肥污水范围的下方边缘处, 只有 4 月 29 日的样品 分布在粪肥污水内 (图 3). 根据 Kendall 等 ${ }^{[33]}$ 的研究, 微生物硝化作用产生的 $\delta^{18} \mathrm{O}_{\text {nitrate }}$ 范围为 $-10 \% \sim 10 \%$. 结合化粪池 $\mathrm{DO}$ 浓度、 $\mathrm{NO}_{3}^{-}$浓度以及 $\delta^{18} \mathrm{O}_{\text {nitrate }}$ 值的范围可以发现 (表 1 、图 3), 其硝酸盐转化过程大部分以硝 化作用为主,这是导致大部分样品 $\delta^{15} \mathrm{~N}_{\text {nitrate }} 、 \delta^{18} \mathrm{O}_{\text {nitrate }}$ 值偏离土壤有机氮和粪肥污水的主要原因.

$1^{\#}$ 落水洞的样品分布在粪肥污水内 (图 3), 表明其硝酸盐来源主要为酒店排放的粪肥污水.

水房泉样品主要分布在土壤有机氮与粪肥污水中 (图 3), 说明整个岩溶地下河系统的硝酸盐来源主要 为酒店粪肥污水和土壤有机氮.

3.2.2 硝酸盐的转化 氮在自然界循环时参与了物理、化学、生物等过程,伴随着产生了同化作用、硝化作用、 反硝化作用等, 这些作用均会导致 $\delta^{15} \mathrm{~N}_{\text {nitrate }} \delta \delta^{18} \mathrm{O}_{\text {nitrate }}$ 发生分馏并改变其特征值 ${ }^{[34-35]}$, 从而使得识别硝酸盐来 源变得困难. 因此, 利用 $\delta^{15} \mathrm{~N}_{\text {nirrate }} \delta \delta^{18} \mathrm{O}_{\text {nitrate }}$ 识别硝酸盐来源的过程中需要评价硝酸盐的转化过程, 才能更好 地为控制硝酸盐输人提供科学依据.

目前,硝酸盐同位素信息是评价硝酸盐转化的有力工具 ${ }^{[36]}$. 研究表明,硝化作用将铵转化成的 $\delta^{15} \mathrm{~N}_{\text {nitrate }}$ 比剩余铵的氮同位素轻,而反硝化作用会造成残留的硝酸盐相对富集 $\delta^{15} \mathrm{~N}_{\text {nirtate }}{ }^{[34]}$. 这一原理具体表现为硝 化作用后 $\mathrm{NO}_{3}^{-}$浓度上升, $\delta^{15} \mathrm{~N}_{\text {nirrate }}$ 值下降; 相反 $\mathrm{NO}_{3}^{-}$浓度下降, $\delta^{15} \mathrm{~N}_{\text {nitrate }}$ 值上升 (反硝化作用) ${ }^{[37]}$, 因此可利用 $\delta^{15} \mathrm{~N}_{\text {nitrate }}$ 与 $\mathrm{NO}_{3}^{-}$的关系识别硝酸盐的部分转化过程. 研究区大多数样品的 $\delta^{15} \mathrm{~N}_{\text {nitrate }}$ 保持在 $3 \% 0 \sim 10 \%$, 而 $\mathrm{NO}_{3}^{-}$ 浓度变化幅度较大 (图 4). 其中, 图 4 右侧化粪池 5 个样品的 $\mathrm{NO}_{3}^{-}$浓度偏高, 但 $\delta^{15} \mathrm{~N}_{\text {nirrate }}$ 低, 表明化粪池发生 


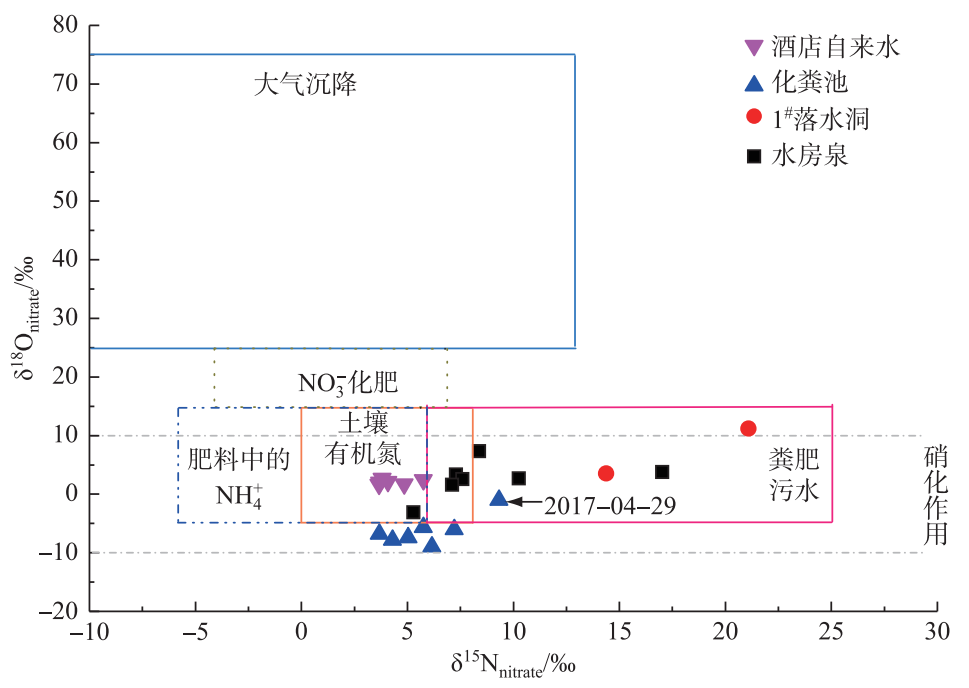

图 3 硝酸盐来源 $\delta^{15} \mathrm{~N}_{\text {nitrate }}$ 与 $\delta^{18} \mathrm{O}_{\text {nitrate }}$ 值范围及研究区硝酸盐氮氧同位素特征值

Fig.3 Ranges of $\delta{ }^{15} \mathrm{~N}_{\text {nitrate }}$ and $\delta{ }^{18} \mathrm{O}_{\text {nitrate }}$ values of potential nitrate sources and characteristic values of nitrate nitrogen-oxygen isotope in the study area

硝化作用, 释放出高浓度的 $\mathrm{NO}_{3}^{-}$(表 1). 然而, 图 4 中 $1^{\#}$ 落水洞样品的 $\delta^{15} \mathrm{~N}_{\text {nitrate }}$ 高, $\mathrm{NO}_{3}^{-}$浓度低, 可与上文水化 学分析共同证明 $1^{\#}$ 落水洞污水发生反硝化作用. 此外, 化粪池 6 月 19 日和 10 月 20 日两个样品、酒店自来水 和水房泉大部分样品的 $\delta^{15} \mathrm{~N}_{\text {nitrate }}$ 与 $\mathrm{NO}_{3}^{-}$浓度介于硝化和反硝化作用之间. 其中, 化粪池 6 月 19 日和 10 月 20 日两个样品的 $\mathrm{NO}_{3}^{-}$浓度相比其他 5 个样品偏低, 主要是采样前景区游客人数减少导致酒店排污量降低所致 (图 2、4). 酒店自来水和水房泉大部分样品的 $\delta^{15} \mathrm{~N}_{\text {nitrate }} 、 \mathrm{NO}_{3}^{-}$浓度较为接近, 可能存在相同的硝酸盐转化过 程. 综上所述, 酒店污水排人化粪池后主要发生硝化作用, 而流经管道进人 $1^{\#}$ 落水洞时发生反硝化作用.

Mariotti ${ }^{[38]} 、$ Kendall $^{[11]}$ 指出在同位素分馏的动力和平衡过程中, 经典瑞利方程能够描述剩余反应物同位 素组成的演化. Chen 等 ${ }^{[39]}$ 的研究证明,运用瑞利方程能够对硝酸盐同位素富集因子进行计算并进一步识别 硝酸盐转化过程. Weng 等 ${ }^{[30]}$ 运用瑞利方程计算同位素富集因子发现近扇硝酸盐转化过程主要为硝化作用, 中扇为同化作用,远扇主要为反硝化作用同时可能发生同化作用. 因此, 基于瑞利方程计算硝酸盐同位素富 集因子的方程可用式 (5) 表示 ${ }^{[30]}$ :

$$
\delta^{15} \mathrm{~N}_{\text {nitrate }}=\delta^{15} \mathrm{~N}_{0 \text { nitrate }}+\varepsilon \ln C\left(\mathrm{NO}_{3}^{-}\right)
$$

式中, $\delta^{15} \mathrm{~N}_{\text {nitrate }}$ 为 $t$ 时刻硝酸盐反应物 $\delta^{15} \mathrm{~N}_{\text {nirrate }}$ 值, $\delta^{15} \mathrm{~N}_{0 \text { nitrate }}$ 为硝酸盐氮同位素的最初组成, $\varepsilon$ 是富集因子, $C\left(\mathrm{NO}_{3}^{-}\right)$是硝酸盐的剩余部分浓度.

通过拟合 $\delta^{15} \mathrm{~N}_{\text {nitrate }}$ 和 $\ln C\left(\mathrm{NO}_{3}^{-}\right)$之间的关系, 计算出化粪池 $\delta^{15} \mathrm{~N}_{\text {nitrate }}=-32.98+10.69 \ln C\left(\mathrm{NO}_{3}^{-}\right)\left(R^{2}=\right.$ $0.25, n=5), 1^{\#}$ 落水洞 $\delta^{15} \mathrm{~N}_{\text {nitrate }}=20.73-8.10 \ln C\left(\mathrm{NO}_{3}^{-}\right)\left(R^{2}=1, n=2\right)$, 酒店自来水 $\delta^{15} \mathrm{~N}_{\text {nitrate }}=19.72-9.72 \ln C$ $\left(\mathrm{NO}_{3}^{-}\right)\left(R^{2}=0.27, n=6\right)$, 水房泉 $\delta^{15} \mathrm{~N}_{\text {nitrate }}=20.54-6.68 \ln C\left(\mathrm{NO}_{3}^{-}\right)\left(R^{2}=0.22, n=7\right)$ (图 4). Kendall 等 ${ }^{[33]}$ 的 研究发现, 反硝化作用导致 $\varepsilon$ 在 $-40 \% 0 \sim-5 \%$ 之间, 同化作用的 $\varepsilon$ 在 $-30 \% 0 \sim 0$ 之间. 除去化粪池 $\mathrm{NO}_{3}^{-}$浓度偏 低的 2 个样品, 拟合其他 5 个样品 $\varepsilon=10.69 \%$. 结合上文水化学与 $\delta^{15} \mathrm{~N}_{\text {nitrate }} \delta \delta^{18} \mathrm{O}_{\text {nitrate }}$ 的分析, 表明其主要发生 强烈的硝化作用导致 $\mathrm{NO}_{3}^{-}$浓度为同期各采样点最高, 这是水房泉岩溶地下河系统最为重要的 $\mathrm{NO}_{3}^{-}$来源之一. $1^{\#}$ 落水洞的 $\varepsilon=-8.10 \%$, 结合上文水化学和 $\delta^{15} \mathrm{~N}_{\text {nitrate }} 、 \delta^{18} \mathrm{O}_{\text {nitrate }}$ 分析发现主要发生反硝化作用, 从而导致该点 $\mathrm{NO}_{3}^{-}$浓度相比其他各点样品处于极低水平. 酒店自来水的 $\varepsilon=-9.72 \%$, 其 $\mathrm{NO}_{3}^{-}$浓度一直稳定在相对较低的水 平,并且 DO 浓度 $>8 \mathrm{mg} / \mathrm{L}$, 表明该点可能发生同化作用. 水房泉的 $\varepsilon=-6.68 \%, 4$ 月 29 日和 8 月 7 日旅游高 峰期样品的 $\delta^{15} \mathrm{~N}_{\text {nitrate }}$ 值、 $\mathrm{NO}_{3}^{-}$浓度分别显著升高, 其余样品的 $\delta^{15} \mathrm{~N}_{\text {nitrate }}$ 值、 $\mathrm{NO}_{3}^{-}$浓度和酒店自来水一样相对较 低且 DO 浓度 $>7 \mathrm{mg} / \mathrm{L}$, 表明硝酸盐转化过程可能与酒店自来水一致, 发生了同化作用. 酒店自来水取自仙 


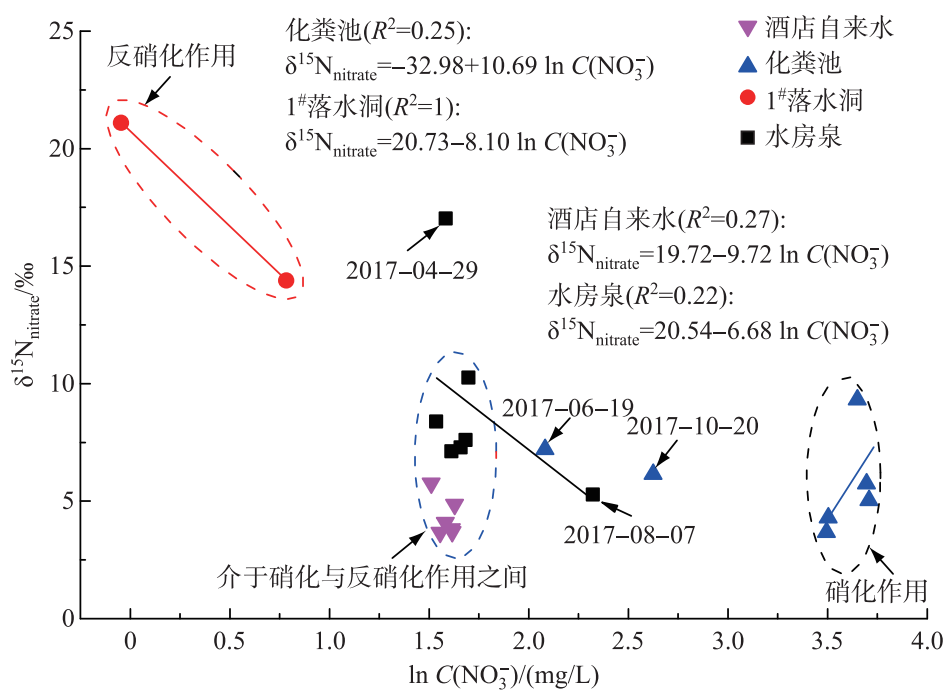

图 $4 \delta^{15} \mathrm{~N}_{\text {nitrate }}$ 与 $\mathrm{NO}_{3}^{-}$浓度的关系

Fig.4 Relationship between $\delta^{15} \mathrm{~N}_{\text {nitrate }}$ and $\mathrm{NO}_{3}^{-}$concentration

女洞,其水源主要为雨水经土壤层和岩溶非饱和带渗滤形成. 水房泉岩溶地下河的水源主要为经土壤层和 岩溶非饱和带渗滤的雨水以及经落水洞直接灌人的酒店污水和雨水, 这一点上文硝酸盐来源分析已充分证 明. 已有监测数据表明, 土壤水 $\mathrm{NO}_{3}^{-}$浓度最高达 $115 \mathrm{mg} / \mathrm{L}$ (未发表), 主要来自土壤有机氮的硝化作用 ${ }^{[40]}$. 高 $\mathrm{NO}_{3}^{-}$浓度的土壤水渗滤至仙女洞、水房泉地下河之后, 其 $\mathrm{NO}_{3}^{-}$浓度基本都保持在 $5 \mathrm{mg} / \mathrm{L}$ 左右 (表 1). 根据野 外实际调查发现, 金佛山生态环境良好, 仙女洞、水房泉周边的岩石上都生长有大片苔藓与草类植物, 因此 结合水化学、硝酸盐来源分析和 $\delta^{15} \mathrm{~N}_{\text {nirrate }}$ 富集因子的计算可判定在运移中发生了同化作用, 这是酒店自来 水、水房泉 $\mathrm{NO}_{3}^{-}$浓度保持在较低水平的重要原因.

基于以上分析可发现, 在拟合 $\delta^{15} \mathrm{~N}_{\text {nitrate }}$ 和 $\ln C\left(\mathrm{NO}_{3}^{-}\right)$关系进行硝酸盐转化过程识别时, 要同时运用水化 学、 $\delta^{15} \mathrm{~N}_{\text {nitrate }} 、 \delta^{18} \mathrm{O}_{\text {nitrate }}$ 分析进行旁证, 才能准确地识别硝酸盐转化过程.

\section{3 基于 SIAR 模型的硝酸盐来源定量解析}

通过硝酸盐来源的定量解析可进一步划分来源的贡献率, 验证 $\delta^{15} \mathrm{~N}_{\text {nitrate }} \delta \delta^{18} \mathrm{O}_{\text {nitrate }}$ 来源定性分析的结 果 ${ }^{[2,5,15,41]}$. 水房泉为研究区岩溶地下河系统的出口, 因此计算水房泉的硝酸盐来源贡献率就可对整个地下 河系统硝酸盐来源进行定量划分. 野外调查中发现, 研究区只有接待游客的酒店, 而无任何工农业活动. 因 此, 本研究将计算的硝酸盐来源设为 3 种, 分别为大气降水、土壤有机氮和粪肥污水. 因未采集本地大气降 水中的 $\delta^{15} \mathrm{~N}_{\text {nitrate }}$ 和 $\delta^{18} \mathrm{O}_{\text {nitrate }}$ 数据, 取与研究区相距 $150 \mathrm{~km}$ 的青木关 ${ }^{[16]}$ 的研究数据替代; 而土壤有机氮与粪 肥污水的 $\delta^{15} \mathrm{~N}_{\text {nitrate }}$ 和 $\delta^{18} \mathrm{O}_{\text {nitrate }}$ 值在各研究中区别不大, 因此采用王静等 ${ }^{[15]}$ 研究中的源数据. 假设模型中分馏 系数 $C_{j k}=0$. 在进行来源贡献率评价时, 应用的是其平均值. 计算结果表明, 水房泉 7 个样品的大气降水、土 壤有机氮和粪肥污水来源的比例分别保持在 $28 \%$ 、36\% 和 36\%左右(图 5).

\section{4 结论与不足}

1) 水房泉的 $\mathrm{NO}_{3}^{-} 、 \mathrm{NH}_{4}^{+} 、 \mathrm{Cl}^{-}$浓度较低, 因游客人数有所波动, 表明该岩溶地下河系统有着良好的自净能 力与生态环境. 空间上, 化粪池 $\mathrm{EC}$ 值和 $\mathrm{NO}_{3}^{-} 、 \mathrm{Cl}^{-}$浓度达到整个岩溶地下河系统最高水平, 而 $\mathrm{DO}$ 浓度达到最 低水平, 污染最为严重. 时间上, 岩溶地下河系统污染程度随游客人数而波动.

2) 水房泉岩溶地下河系统硝酸盐的重要来源为土壤有机氮与粪肥污水. 其中, 酒店自来水、水房泉地下 水的硝酸盐转化过程以同化作用为主, 化粪池污水以硝化作用为主, $1^{\#}$ 落水洞污水出现反硝化作用.

3) 水房泉岩溶地下河系统硝酸盐来源贡献率中, 土壤有机氮的贡献率约为 $36 \%$, 粪肥污水的贡献率约 


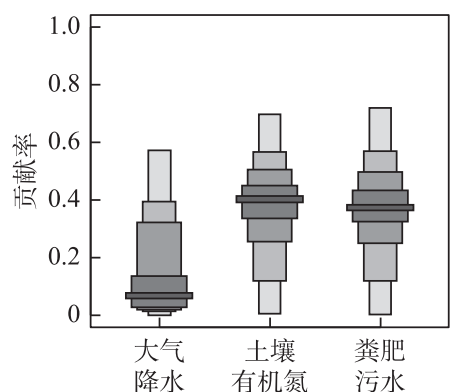

(a) 2017-04-29

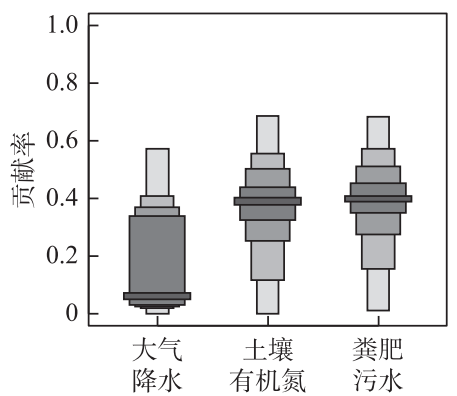

(d) 2017-07-12

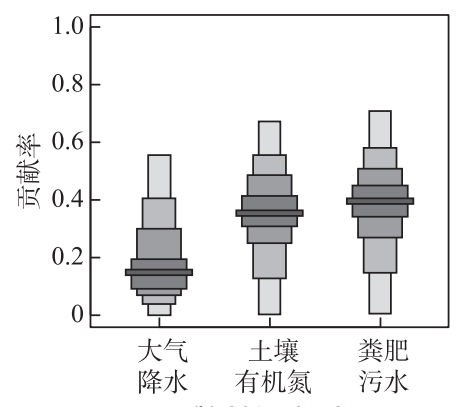

(b) 2017-05-25

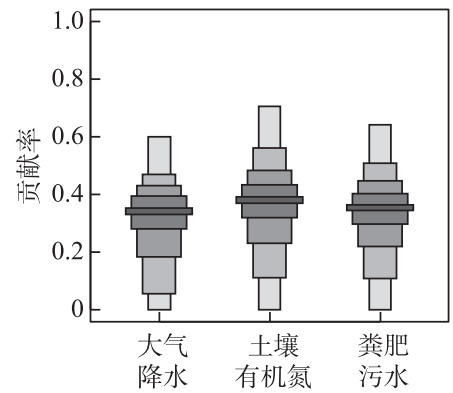

(e) 2017-08-07

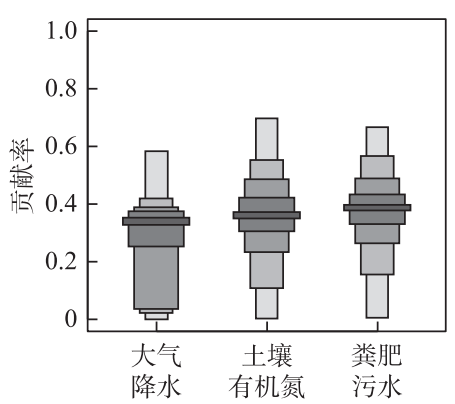

(g) 2017-10-20

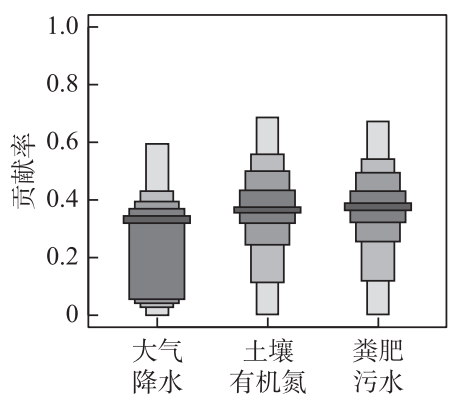

(c) 2017-06-19

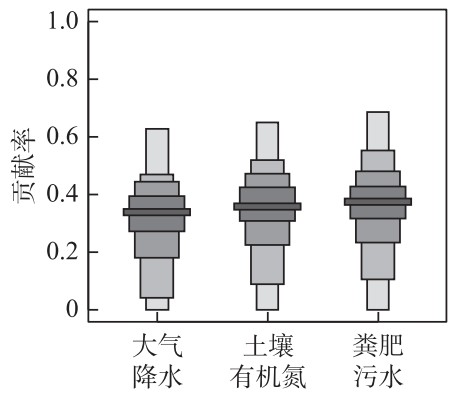

(f) 2017-08-28

图 5 基于 SIAR 模型的硝酸盐贡献率(从浅到深箱型图依次表示 5\%、25\%、50\%、75\%、95\%)

Fig.5 Nitrate contribution rate based on SIAR model in which boxplots representing the $5 \%, 25 \%, 50 \%, 75 \%$ and $95 \%$ from light to dark

为 $36 \%$. 虽然水房泉岩溶地下河系统有着良好的自净能力与生态环境, 但随着游客人数的上升硝酸盐污染 程度必定会不断上升. 因此, 景区应适当控制游客人数,并采取生物措施和工程措施综合治理硝酸盐污染.

未来硝酸盐转化的相关研究还需要检测硝化、反硝化细菌进一步证明, 本研究受限于当前研究条件未 能检测.

致谢: 感谢西南大学赵珍、谢国文、詹兆君、马哲显、陈峰、付伟、任问䈥、田宁等硕士研究生在野外、实验方面 给予的帮助.

\section{5 参考文献}

[ 1 ] Ma ZF, Yang Y, Lian XY et al. Identification of nitrate sources in groundwater using a stable isotope and 3DEEM in a landfill in Northeast China. Science of the Total Environment, 2016, 563/564: 593-599.

[ 2 ] Ding JT, Xi BD, Gao RT et al. Identifying diffused nitrate sources in a stream in an agricultural field using a dual isotopic approach. Science of the Total Environment, 2014, 484: 10-18. 
[ 3 ] Yang PH, Li Y, Groves C et al. Coupled hydrogeochemical evaluation of a vulnerable karst aquifer impacted by septic effluent in a protected natural area. Science of the Total Environment, 2019, 658: 1475-1484.

[ 4 ] Xue D, Botte J, Baets BD et al. Present limitations and future prospects of stable isotope methods for nitrate source identification in surface and groundwater. Water Research, 2009, 43: 1159-1170.

[ 5 ] Xue D, Baets BD, Cleemput OV et al. Use of a Bayesian isotope mixing model to estimate proportional contributions of multiple nitrate sources in surface water. Environmental Pollution, 2012, 161: 43-49.

[6] Fan AM, Steinberg VE. Health implications of nitrate and nitrite in drinking water: an update on methemoglobinemia occurrence and reproductive and developmental toxicity. Regulatory Toxicology \& Pharmacology, 1996, 23: 35-43.

[ 7 ] Almasri MN. Nitrate contamination of groundwater: a conceptual management framework. Environmental Impact Assessment Review, 2007, 27 : 220-242.

[ 8 ] Kohl DH, Shearer GB, Commoner B. Fertilizer nitrogen: contribution to nitrate in surface water in a corn belt watershed. Science, 1971, 174: 1331-1334.

[ 9 ] Wang DS. Basis for the use of nitrogen isotopes to identify nitrogen contamination of groundwater. Acta Geoscientia Sinica, 1997, 18 (2) : 220-223. [王东升. 氮同位素比 $\left({ }^{15} \mathrm{~N} /{ }^{14} \mathrm{~N}\right)$ 在地下水氮污染研究中的应用基础. 地球学报, 1997,18 (2) : 220-223.]

[10] Shen ZL, Zhu WH, Zhong ZS eds. Basic course for hydrographic geochemistry. Beijing: Geology Press, 1993: 137-140. [沈照理, 朱宛华, 钟佐楽. 水文地球化学基础. 北京: 地质出版社, 1993: 137-140.]

[11] Kendall C. Tracing nitrogen sources and cycling in catchments. Isotope Tracers in Catchment Hydrology, 1998: 522-552.

[12] Liu CQ, Li SL, Lang YC et al. Using $\delta^{15} \mathrm{~N}$ and $\delta^{18} \mathrm{O}$-values to identify nitrate sources in karst ground water, Guiyang, Southwest China. Environmental Science \& Technology, 2006, 40: 6928-6933.

[13] Wang ZJ, Yang PH, Kuang YL et al. Temporal and spatial variations of the nitrate-nitrogen sources in an underground river using ${ }^{15} \mathrm{~N}$ isotope technique. Environmental Science, 2009, 30(12):3548-3554. [汪智军, 杨平恒, 旷顷金等. 基 于 ${ }^{15} \mathrm{~N}$ 同位素示踪技术的地下河硝态氮来源时空变化特征分析. 环境科学, 2009, 30(12) : 3548-3554.]

[14] Xu ZW, Zhang XY, Ren YF et al. Spatial changes and sources of nitrate in Beijing urban ecosystem surface water. Environmental Science, 2012, 33(8) : 2569-2573. [徐志伟, 张心昱, 任玉芬等. 北京城市生态系统地表水硝酸盐污染空 间变化及其来源研究. 环境科学, 2012, 33(8) : 2569-2573.]

[15] Wang J, Ye Y, Wang YQ et al. Using $\delta^{15} \mathrm{~N}$ and $\delta^{18} \mathrm{O}$ values to identify sources of nitrate in the Dianbu River in the Chaohu Lake Basin. Journal of Hydraulic Engineering, 2017, 48(10): 1195-1205. [王静, 叶寅, 王允青等. 利用氮氧同位 素示踪技术解析巢湖支流店埠河硝酸盐污染源. 水利学报,2017, 48(10): 1195-1205.]

[16] Sheng T, Yang PH, Xie GW et al. Nitrate-nitrogen pollution sources of an underground river in karst agricultural area u$\operatorname{sing} \delta^{15} \mathrm{~N}$ and $\delta^{18} \mathrm{O}$ isotope technique. Environmental Science, 2018, 39(10): 4547-4555. [盛婷, 杨平恒, 谢国文等. 基于 $\delta^{15} \mathrm{~N}$ 和 $\delta^{18} \mathrm{O}$ 的农业区地下河硝酸盐污染来源. 环境科学, 2018, 39(10)：4547-4555.]

[17] Yuan DX, Jiang YJ, Shen LC et al eds. Modern karstology. Beijing: Science Press, 2016: 293. [袁道先, 蒋勇军, 沈立 成等. 现代岩溶学. 北京: 科学出版社, 2016: 293.]

[18] Yang PH, Zhan ZJ, Ming XX et al. Hydrochemical variation of the karst groundwater impacted by the contaminant discharge from a tourism hotel. $J$ Lake Sci, 2019, 31(2): 416-428. DOI: 10.18307/2019.0211. [杨平恒, 詹兆君, 明晓 星等. 旅游酒店排污影响下的岩溶地下水水化学变化. 湖泊科学, 2019, 31(2): 416-428.]

[19] Committee of Jinfoshan Management, Nanchuan District, Chongqing. The 2017 Work Report of Ticket Management of Scenic Area in Jinfoshan, 2018. [金佛山风景名胜区 2017 年门票管理工作报告. 重庆市南川区金佛山管理委员 会, 2018.]

[20] Wu KY, Wang P, Shen LC et al. Spatial and temporal variability of nitrate contaminant in groundwater in Jinfo Mt. Area, Chongqing, China. Environmental Science, 2011, 32(11):3247-3254. [伍坤宇, 王鹏, 沈立成等. 金佛山地区地下水 硝态氮污染时空变异性研究. 环境科学, 2011, 32(11) : 3247-3254.]

[21] Deng HP, Cheng K, Wang JB et al eds. The brief development history of national nature reserves in Jinfoshan, Chongqing. Chongqing: Southwest China Normal University Press, 2015: 1-3. [邓洪平, 程科, 王军波等. 重庆金佛山国家级自然 保护区发展简史. 重庆: 西南师范大学出版社, 2015: 1-3.]

[22] Li LL, Kuang MS, Jiang YJ et al. Study on the karst ecosystem of Mountain Jinfo. Journal of Sichuan Normal University: Natural Science, 2003, 26(2) : 201-204. [李林立, 况明生, 蒋勇军等. 金佛山岩溶生态系统初步探讨一一岩溶泉 
水化学特征分析. 四川师范大学学报：自然科学版, 2003, 26(2) : 201-204.]

[23] Wu YX. Hydrologic modeling of karst spring using storm water management model [Dissertation]. Chongqing: Southwest University, 2008. [吴月霞. 基于 SWMM 的岩溶泉域水文过程的模拟研究 [学位论文]. 重庆: 西南大学, 2008.]

[24] Yu ZL, Yuan DX, Yang PH et al. Influences of tourism activities on hydrochemistry of karst groundwater revealed by principal component analysis and on-line monitoring technique. Acta Geoscientica Sinica, 2016, 37(2) : 232-240. [于正良, 袁道先, 杨平恒等. 基于 PCA 和在线监测技术研究旅游活动对岩溶地下水水化学的影响. 地球学报, 2016, 37 (2) : 232-240.]

[25] Yang PH, Ming XX, Groves C et al. Impact of hotel septic effluent on the Jinfoshan Karst aquifer, SW China. Hydrogeology Journal, 2019, 27: 321-334.

[26] Parnell AC, Inger R, Bearhop S et al. Source partitioning using stable isotopes: coping with too much variation. PLoS One, 2010, 5(3): 1-5.

[27] Zhang T. Dynamic changes and influencing factors of nitrate in a surface stream in subtropical karst area, SW China [Dissertation]. Chongqing: Southwest University, 2015. [张陶. 典型岩溶区溪流中硝酸盐动态变化及其影响因素研究 [学位论文]. 重庆: 西南大学, 2015.]

[28] Wu YG. Denitrification in groundwater systems. Techniques and Equipment for Environmental Pollution Control, 2002, 3 (3) : 27-31. [吴耀国. 地下水环境中反硝化作用. 环境污染治理技术与设备，2002，3(3) : 27-31.]

[29] Silva SR, Ging PB, Lee RW et al. Forensic applications of nitrogen and oxygen isotopes in tracing nitrate sources in urban environments. Environmental Forensics, 2002, 3: 125-130.

[30] Weng TN, Liu CW, Kao YH et al. Isotopic evidence of nitrogen sources and nitrogen transformation in arsenic-contaminated groundwater. Science of the Total Environment, 2017, 578: 167-185.

[31] Nestler A, Berglund M, Accoe F et al. Isotopes for improved management of nitrate pollution in aqueous resources: review of surface water field studies. Environmental Science \& Pollution Research, 2011, 18: 519-533.

[32] Miao Y, Zhang C, Xiao Q et al. Dynamic variations and sources of nitrate during dry season in Lijiang River. Environmental Science, 2018, 39(4) : 1589-1597. [苗迎, 章程, 肖琼等. 漓江段地表水体旱季硝酸盐动态变化特征及其来源. 环境科学, 2018, 39(4): 1589-1597.]

[33] Kendall C, Elliott EM, Wankel SD. Tracing anthropogenic inputs of nitrogen to ecosystems//Michener RH, Lajtha K eds. Stable Isotopes in Ecology \& Environmental Science. Blackwell Pub, 2007: 375-449.

[34] Chen FJ, Li XH, Jia GD. The application of nitrogen and oxygen isotopes in the study of nitrate in rivers. Advances in Earth Science, 2007, 22(12)：1251-1257. [陈法锦, 李学辉, 贾国东. 氮氧同位素在河流硝酸盐研究中的应用. 地 球科学进展, 2007, 22(12): 1251-1257.]

[35] Mao W, Liang ZW, Li W et al. Research advances in identifying nitrate pollution sources of water environment by using nitrogen and oxygen stable isotopes. Chinese Journal of Applied Ecology, 2013, 24(4) : 1146-1152. [毛巍, 梁志伟, 李伟 等. 利用氮、氧稳定同位素识别水体硝酸盐污染源研究进展. 应用生态学报, 2013, 24(4) : 1146-1152.]

[36] Li C, Li SL, Yue FJ et al. Identification of sources and transformations of nitrate in the Xijiang River using nitrate isotopes and Bayesian model. Science of the Total Environment, 2019, 646: 801-810.

[37] Cao WZ, Huang Z, Zhai WD et al. Isotopic evidence on multiple sources of nitrogen in the northern Jiulong River, Southeast China. Estuarine Coastal \& Shelf Science, 2015, 163: 37-43.

[38] Mariotti A, Landreau A, Simon B. ${ }^{15} \mathrm{~N}$ isotope biogeochemistry and natural denitrification process in groundwater: application to the chalk aquifer of northern France. Geochimica et Cosmochimica Acta, 1988, 52: 1869-1878.

[39] Chen FJ, Jia GD, Chen JY. Nitrate sources and watershed denitrification inferred from nitrate dual isotopes in the Beijiang River, south China. Biogeochemistry, 2009, 94: 163-174.

[40] Yang PH, Yuan DX, Ren YR et al. Nitrate storage and transport within a typical karst aquifer system in the paralleled ridge-valley of east Sichuan. Environmental Science, 2012, 33(9): 3124-3131. [ 杨平恒, 袁道先, 任幼蓉等. 川东平行 岭谷区典型岩溶含水系统中 $\mathrm{NO}_{3}^{-}$的存储和运移. 环境科学, 2012, 33(9): 3124-3131.]

[41] Lu Y. The study on nitrogen sources and migration which is based on isotopic analysis method [Dissertation]. Chongqing: Chongqing Jiaotong University, 2013. [卢义. 基于同位素分析法的氮来源及迁移过程研究 [ 学位论文]. 重庆: 重庆 交通大学, 2013.] 